ARTICLE

\title{
Structural basis of transcobalamin recognition by human CD320 receptor
}

Amer Alam¹, Jae-Sung Woo ${ }^{1}$, Jennifer Schmitz ${ }^{1}$ Bernadette Prinz ${ }^{1}$, Katharina Root ${ }^{2}$, Fan Chen², Joël S. Bloch¹, Renato Zenobi ${ }^{2} \&$ Kaspar P. Locher ${ }^{1}$

Cellular uptake of vitamin B12 (cobalamin) requires capture of transcobalamin (TC) from the plasma by CD320, a ubiquitous cell surface receptor of the LDLR family. Here we present the crystal structure of human holo-TC in complex with the extracellular domain of CD320, visualizing the structural basis of the TC-CD320 interaction. The observed interaction chemistry can rationalize the high affinity of CD320 for TC and lack of haptocorrin binding. The in vitro affinity and complex stability of TC-CD320 were quantitated using a solid-phase binding assay and thermostability analysis. Stable complexes with TC were also observed for the disease-causing CD320 $\triangle \mathrm{E} 88$ mutant and for the isolated LDLR-A2 domain. We also determined the structure of the TC-CD320 $\mathrm{E} 88$ complex, which revealed only minor changes compared with the wild-type complex. Finally, we demonstrate significantly reduced in vitro affinity of $\mathrm{TC}$ for $\mathrm{CD} 32 \mathrm{O}$ at low $\mathrm{pH}$, recapitulating the proposed ligand release during the endocytic pathway. 
M ammals depend on dietary uptake of Cobalamin (Cbl), which is a cofactor of at least two enzymes that are part of essential biochemical pathways ${ }^{1,2}$. A complex uptake system involving several transport proteins and cellular receptors facilitates efficient transport of this scarce vitamin ${ }^{3-10}$. Defects at any stage of this process have been found to lead to Cbl deficiency and severe developmental defects in patients ${ }^{11,12}$. transcobalamin (TC)-bound $\mathrm{Cbl}$ is transported into cells by receptor-mediated endocytosis, which requires $\mathrm{Ca}^{2+}$-dependent complex formation of TC with its cognate cell surface receptor CD320 (ref. 13). CD320 is a member of the Low-density Lipoprotein Receptor (LDLR) family ${ }^{13,14}$, a large group of mostly multi-ligand receptors involved in cholesterol uptake and other physiologically essential functions ${ }^{15,16}$. CD320 features an N-terminal, extracellular fragment comprising two LDLR type A (LDLR-A) domains separated by an epidermal growth factor (EGF) homology domain, the exact function of which has so far not been determined. It also contains a single transmembrane helix and a C-terminal cytoplasmic region involved in complex internalization ${ }^{8}$. While the majority of related LDLR domaincontaining receptors bind multiple and distinct ligands ${ }^{17}, \mathrm{CD} 320$ has been reported to bind TC with high specificity and affinity as evidenced by the lack of interference in TC binding from related LDLR ligands like LDL and RAP as well as high affinity for TC of both the full-length receptor and its soluble ectodomain ${ }^{8,13,18}$. Even haptocorrin ( $\mathrm{HC})$, a high affinity Cbl-binding protein that shares significant sequence and structural similarities with TC and is also circulating in the plasma, is not recognized by CD320.

Although several of the proteins facilitating Cbl uptake and transport from saliva to the blood stream have been structurally characterized ${ }^{19-22}$, the final uptake step into cells has not been visualized at a molecular level. Such insight is required not only to understand the basis of specific TC-Cbl uptake into cells, but also to rationalize disease-causing mutations that interfere with this process. Furthermore, expression of CD320 is reported to be highest in actively proliferating cells and is strongly upregulated in many cancer cells, prompting interest in its potential targeting through receptor directed drug delivery ${ }^{23,24}$ or its exploitation for tumour diagnostics. In light of its physiological relevance, we set out to biochemically and structurally characterize the TC-CD320 complex, revealing key molecular details of the high affinity interaction, its regulation by $\mathrm{pH}$ and the in vitro effect of mutations of $\mathrm{CD} 320$ on its interaction with TC.

\section{Results}

Structural and functional analysis of TC-CD320 complex. The challenges associated with the production of suitable amounts of homogenous, stable, and functional CD320 protein have so far prevented its structural analysis $7,25,26$. To overcome these challenges, we heterologously co-expressed human TC and the external, TC-binding part of CD320 in secreted form in baculovirus-infected insect cells, taking advantage of the enhanced biochemical stability of the complex over individual components. CD320 was N-terminally fused to maltose-binding protein (MBP) that was subsequently cleaved on purification. We purified the TC-CD320 complex to homogeneity in the presence of excess cyanocobalamin (Cbl-CN) to ensure full occupancy of the Cbl-binding site. The obtained complex had a stoichiometry of $1: 1$ as confirmed by MALDI mass spectrometry (Supplementary Fig. 1).

The stability of the TC-CD320 complex was analysed by determining its thermostability upon incubation at increasing temperatures, followed by size exclusion chromatography (SEC) analysis. As shown in Fig. 1a, we observed a $>20^{\circ} \mathrm{C}$ drop in the apparent Tm in the presence of the $\mathrm{Ca}^{2+}$ chelator ethylene glycol tetraacetic acid (EGTA), confirming the stabilizing effect of $\mathrm{Ca}^{2+}$ on the TC-CD320 complex. We developed an enzyme-linked immunosorbent assay-based solid-phase binding assay to determine the affinity of the TC-CD320 interaction by using immobilized MBP-tagged, biotinylated CD320 (CD320 $\left.{ }_{\mathrm{AVI}}\right)$. Tag-less TC was obtained by EGTA induced dissociation of a pre-formed TC-CD $320_{\mathrm{AVI}}$ complex immobilized on streptavidin resin. TC binding was quantitated using a commercial monoclonal antibody (A-5) targeting a distinct C-terminal epitope of TC, away from its receptor-binding site. As shown in Fig. 1b, TC was confirmed to bind to CD320 with high affinity $\left(K_{\mathrm{D}} \sim 1.5 \mathrm{nM}\right)$, in line with the high thermal stability of the complex.

To analyse whether a single LDLR-A domain of CD320 was capable of binding TC (analogous to observations with the Reelin/LDLR-A1 complex ${ }^{27}$ ), we individually co-expressed each domain with TC. Complex formation was observed both for LDLR-A1 and LDLR-A2, which is in contrast to a previous suggestion that two LDLR-A modules are the minimal binding unit for TC (ref. 8). We found that a complex containing only LDLR-A2 had an apparent Tm only $\sim 5^{\circ} \mathrm{C}$ lower than the equivalent complex containing full-length CD320, and a dissociation constant of $\sim 10 \mathrm{nM}$, slightly reduced compared to full-length CD320 (Fig. 1b). In contrast, the complex with LDLR-A1 appeared less stable on purification, indicating a decreased stability and precluding a detailed thermostability or solid-phase binding analysis.

Various CD320 truncations were generated and tested and a final crystallization construct comprising residues 53-199 of the extracellular domain of CD320 and TC residues 1-409 was chosen for structure determination. The TC-CD320 co-crystal structure, phased by molecular replacement using Cbl-bound TC as a search model, was refined to $2.1 \AA$ resolution and revealed a 1:1 complex of TC-CD320 (Fig. 1c). Whereas clear electron density was observed for LDLR-A1 residues 53-89 and LDLR-A2 residues 129-171, no density was observed for the EGF-like domain (residues 90-128). Both LDLR-A domains and TC contain several disulfide bridges, the positions of the majority of which were confirmed using an anomalous difference map calculated from data collected at a wavelength of $1.6 \AA$ (Table 1 ). In the same map, $\mathrm{Ca}^{2+}$ ions bound to the LDLR-A domains and the Cobalt of Cbl bound to TC were identified.

Overall architecture of the TC-CD320 complex. In agreement with epitope mapping studies ${ }^{28}$, both LDLR-A1 and LDLR-A2 of CD320 exclusively interact with the $\alpha$ domain of TC (Fig. 1c). The structure of CD320-bound TC is very similar to that of its free state ${ }^{20}$ (r.m.s.d of all $\mathrm{C} \alpha$ atoms of $0.43 \AA$, Supplementary Fig. 2) with a few key differences. First, we observed electron density for a fourth disulfide bridge linking residues Cys65-Cys78, both of which are conserved in several primate and rodent species ${ }^{20}$. Second, our structure revealed clear electron density for the loop connecting the $\alpha$ and $\beta$ domains of TC. Finally, we observed electron density for the loop carrying the Cbl-binding His173.

To confirm the implications of the observed interface, we co-expressed CD320 with a truncated version of TC containing only its $\alpha$ domain (TC $\alpha$ ) to mimic its apo form. We expressed the $\mathrm{TC} \alpha-\mathrm{CD} 320$ complex in insect cells to avoid denaturing conditions previously used to obtain apo-TC (ref. 29). We could indeed purify a TC $\alpha-\mathrm{CD} 320$ complex with a $1: 1$ stoichiometry, which exhibited a decreased stability (apparent $\mathrm{Tm}$ of $\sim 60^{\circ} \mathrm{C}$ ) compared with the complex containing fulllength TC (apparent Tm of $\sim 70^{\circ} \mathrm{C}$, Fig. 1a). 
a

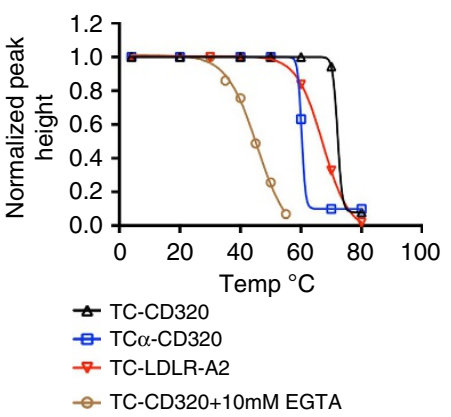

b

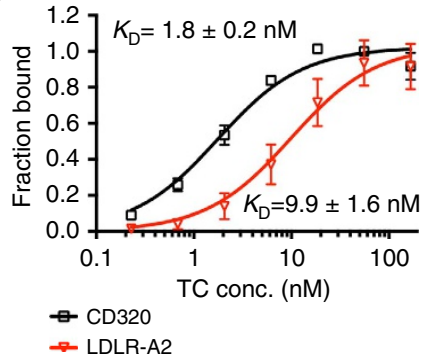

C

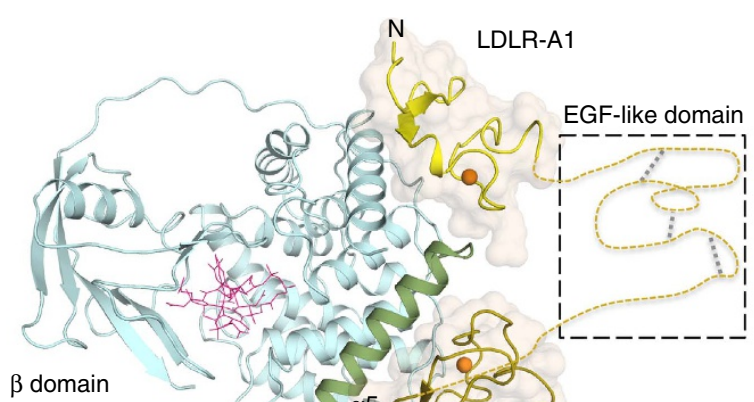

LDLR-A2

Plasma membrane $\alpha$ domain

Internalization sequence

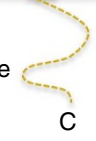

Figure 1 | Characterization of the TC-CD320 complex. (a) Thermostability curves for different TC-CD320 complexes, demonstrating decreased complex stability in the presence of EGTA as well as for the TC $\alpha$-CD320 and TC-LDLR-A2 complexes. (b) Solid-phase binding assay quantitating TC binding to full-length CD320 (black) and to LDLR-A2 (red; $n=5$, error bars indicate S.D). (c) Overall structure of TC-CD320 complex showing TC (cyan) and LDLR-A1 and LDLR-A2 of CD320 (yellow and gold, respectively). The likely position of the EGF-like module (boxed) bridging the two LDLR-A domains is shown schematically. The transmembrane domain (yellow cylinder) and internalization motif of CD320 were not part of the expression construct. Orange spheres represent bound $\mathrm{Ca}^{2+}$ ions. Bound $\mathrm{Cbl}$, sandwiched between the $\alpha$ and $\beta$ domains of TC, is shown in pink and TC helix $\alpha 5$ is coloured green.

\section{Table 1 | Data collection and refinement statistics.}

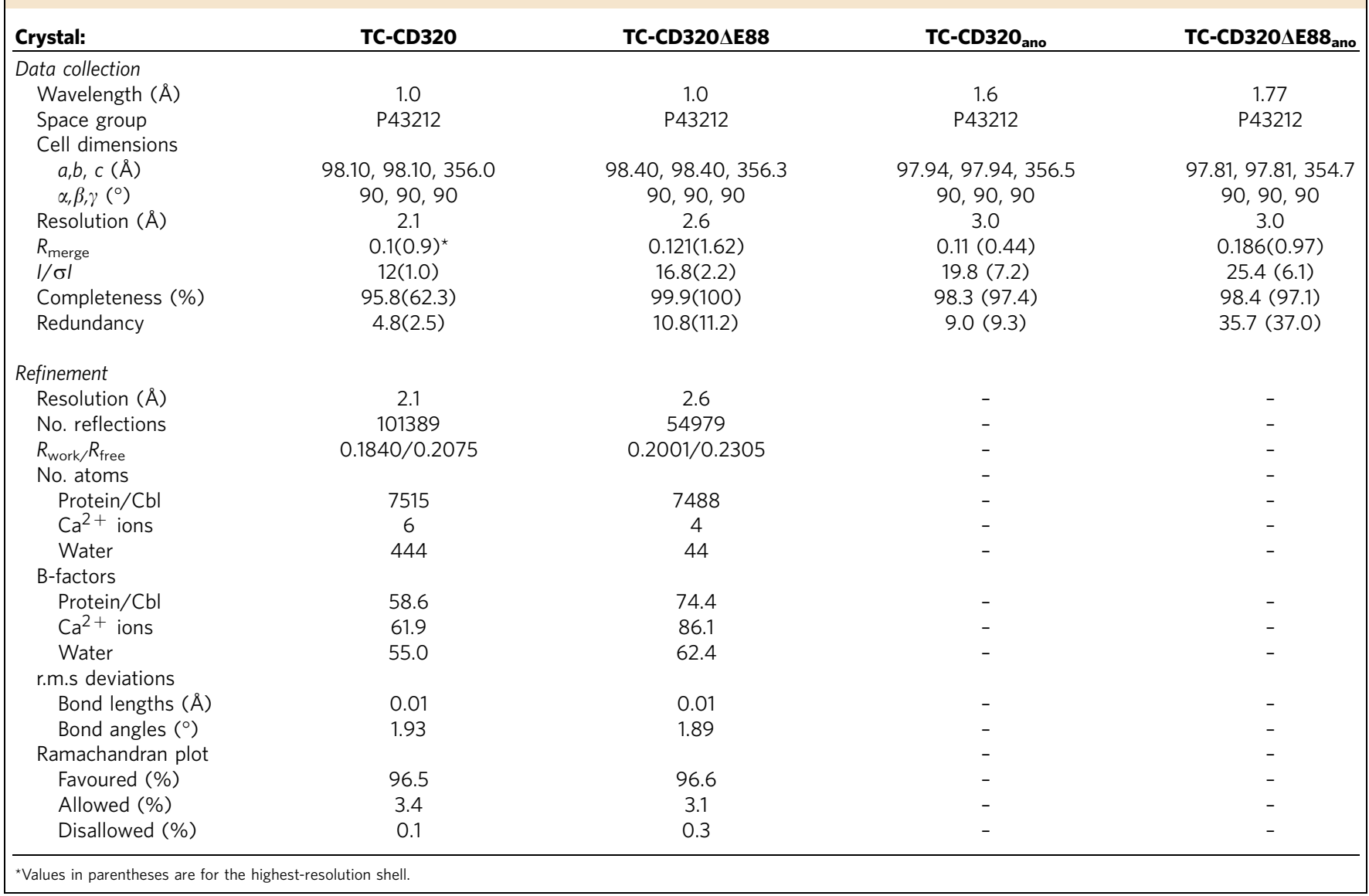


The interface of TC and CD320 consists of $\sim 900 \AA^{2}$ buried surface area, with LDLR-A2 contributing $486 \AA^{2}$ compared with $405 \AA^{2}$ for LDLR-A1. The fold of the LDLR-A domains of CD320 is similar to that of related family members ${ }^{30-34}$ (Fig. 2a-c). Both LDLR-A domains contain central $\mathrm{Ca}^{2+}$ ions bound by four conserved acidic residues and two backbone carbonyls in an octahedral coordination. They also contain a PLxWRCD motif that is conserved in other CD320 orthologs and related proteins ${ }^{13}$. The backbone carbonyl of the tryptophan residue in this motif (Trp72 and Trp150 in LDLR-A1 and LDLR-A2, respectively) coordinates the central $\mathrm{Ca}^{2+}$ ion, while its side chain is stabilized through a stacking interaction with the preceding proline residue. In the case of LDLR-A2, this interaction is further stabilized through stacking with Trp115 and hydrogen bonding with Glu118 of TC. These latter interactions are absent in the LDLR-A1/TC interface. In LDLR-A1, the $\mathrm{Ca}^{2+}$-coordinating carboxylate groups of Asp75 and Asp79 form salt bridges with Lys105 of TC, forming an 'acidic necklace' previously found to be a key feature of LDLR/ ligand complexes ${ }^{32}$ (Fig. 2a). The equivalent acidic necklace in LDLR-A2 is formed by Lys114 of TC and the $\mathrm{Ca}^{2+}$ coordinating carboxylate groups of Asp153 and Asp157 of CD320 (Fig. 2b).

A network of disulfide linkages stabilizes the folds of both LDLR-A1 and LDLR-A2, with disulfide formation following the 1-3/2-5/4-6 pattern observed previously ${ }^{30-32,34,35}$. LDLR-A1 contacts TC helices $\alpha 3$ and $\alpha 5$, whereas LDLR-A2 interacts with TC helix $\alpha 5$ and the loop between helices $\alpha 6$ and $\alpha 7$ (Fig. 2b). LDLR-A2 is involved in an extensive network of electrostatic and hydrogen-bonding interactions with TC helix $\alpha 5$ (Fig. 2b) including an additional salt bridge between TC2 residue Arg122 and Glu136 of LDLR-A2. The overall quality of electron density in LDLR-A2 is higher than that of LDLR-A1. This is especially apparent for the bound $\mathrm{Ca}^{2+}$ ion and $\mathrm{Ca}^{2+}$-coordinating residues and disulfide bond pairs. We also observe higher average B-factors for bound $\mathrm{Ca}^{2+}$ ions in LDLRA1 compared with LDLR-A2 ( $\sim 80 \AA^{2}$ and $44 \AA^{2}$, respectively). Overall these results indicate increased motion/conformational disorder in LDLR-A1 compared with LDLR-A2.

Dependence of TC-CD320 binding affinity on $\mathrm{pH}$. Ligand interactions of the LDLR family members are thought to be modulated by histidine residues located at the binding interfaces and responding to changes in $\mathrm{pH}$, a mechanism termed the 'histidine switch'16,36-39. An analysis of the TC-CD320 interface revealed three histidine residues from TC (His56, His104 and His154) and one from CD320 (His155) that may play a role in modulating the interaction (Fig. 3a). Among the observed interactions, the contact between His104 of TC and Asp77 of LDLR-A1 is notable because the backbone carbonyl of Asp77 coordinates the central $\mathrm{Ca}^{2}+$ ion. Furthermore, the backbone carbonyl of the interface residue His155 of CD320 also coordinates the central $\mathrm{Ca}^{2+}$ ion in LDLR-A2. This histidine also interacts with Asp157 of LDLR-A2, one of the acidic necklace forming residues. The above suggests that an intricate structural link exists between the $\mathrm{pH}$-dependent interface and the $\mathrm{Ca}^{2}{ }^{+}$-coordination in the LDLR-A2 domain. Finally, His104 of TC is in a special location where it could interact with residues from both LDLR-A1 and LDLR-A2.

We analysed the effect of changes in $\mathrm{pH}$ on the TC-CD320 interaction by incubating an immobilized complex of TC and biotionylated receptor $\left(\mathrm{CD} 320_{\mathrm{AVI}}\right)$ at $\mathrm{pH} 4.8$ and $\mathrm{pH} 7.5$ followed by SDS-polyacrylamide gel electrophoresis analysis. We observed a release of TC from the complex at low, but not at physiological $\mathrm{pH}$ (Fig. 3b). We quantitated this effect using our solid-phase binding assay by comparing binding of isolated tag-less TC to immobilized $\mathrm{CD} 320_{\mathrm{AVI}}$ at $\mathrm{pH} 4.8$ and $\mathrm{pH}$ 7.5. As shown in Fig. 3c, the affinity of TC for CD320 is significantly reduced at lower $\mathrm{pH}\left(K_{\mathrm{D}} \sim 1.0 \mathrm{nM}\right.$ at $\mathrm{pH} 7.5$ and $\sim 58 \mathrm{nM}$ at $\left.\mathrm{pH} 4.8\right)$.

Structure of the TC-CD320 $\Delta$ E88 complex. Deletion of a single glutamate $(\triangle \mathrm{E} 88)$ in LDLR-A1 of CD320 has been associated with $\mathrm{Cbl}$ deficiency observed in patients carrying the mutation ${ }^{40}$. To investigate whether a structural effect was underpinning this defect, we generated a complex of TC with CD320 $\Delta \mathrm{E} 88$, characterized it biochemically and determined its structure at $2.6 \AA$ resolution (Supplementary Fig. 3). Protein expression and purification were near identical to the wild-type complex. However, the purified complex displayed increased propensity to form non-specific multimers as analysed by SEC (Supplementary Fig. 4), requiring an additional SEC purification step for crystallization. As the mutant complex structure was of lower resolution than that of the wild type, we confirmed the positions of disulfide bonds and bound $\mathrm{Ca}^{2}+$ ions by using anomalous difference peaks from data collected at a wavelength of $1.77 \AA$.

The TC-CD320 $\Delta$ E88 structure revealed a similar domain arrangement and binding interface as the wild-type protein
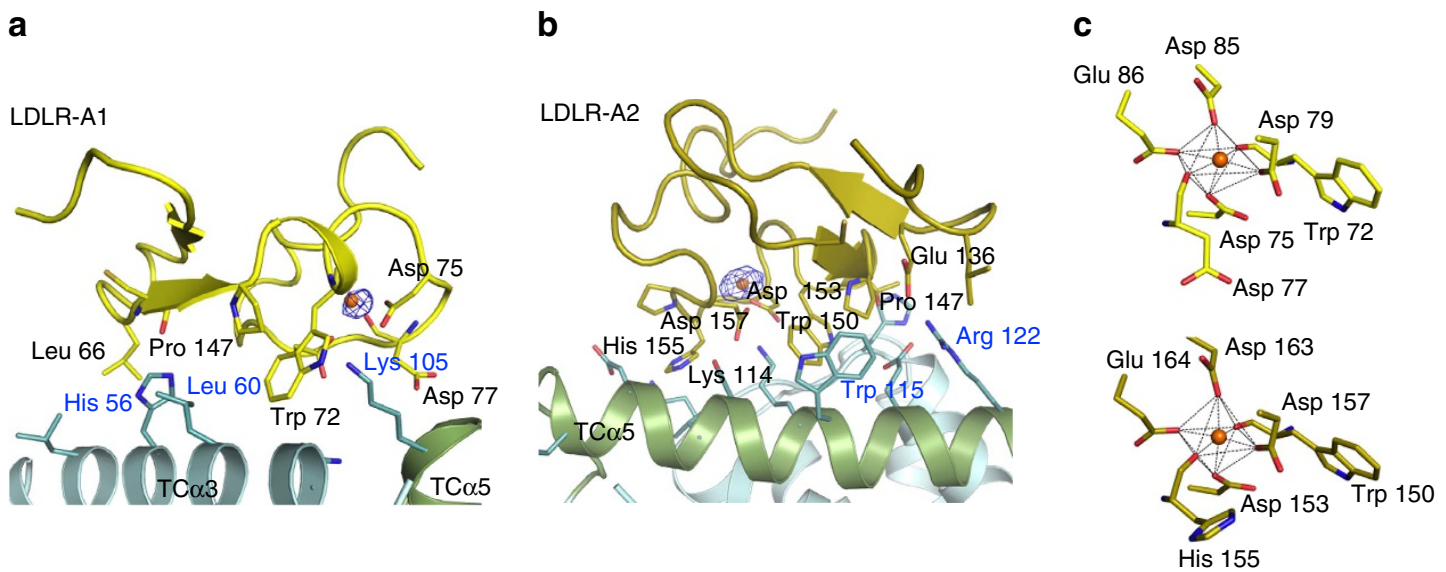

Figure 2 | Details of the TC-CD320 binding interface. (a) Interactions of LDLR-A1 and (b) LDLR-A2 with TC. TC helix $\alpha 5$ is coloured green. Ca ${ }^{2+}$ ions are indicated as orange spheres with corresponding anomalous difference density peaks shown (blue mesh contoured at $4 \sigma$ ). TC and CD320 residues involved in intermolecular contacts and $\mathrm{Ca}^{2+}$ coordination are shown as sticks and labelled blue and black, respectively. (c) Close-up views showing octahedral $\mathrm{Ca}^{2}+$ coordination in LDLR-A1 (yellow, top) and LDLR-A2 (gold, bottom). 
a

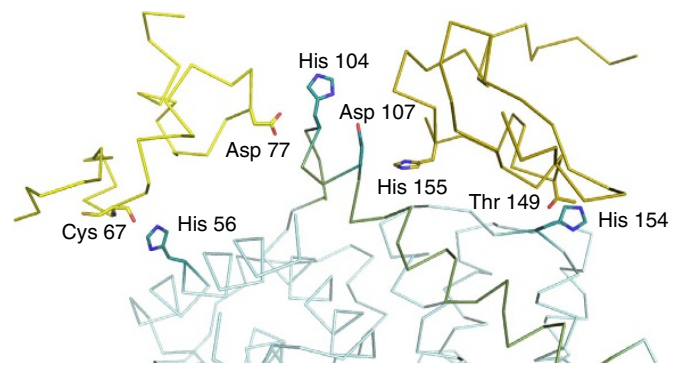

b

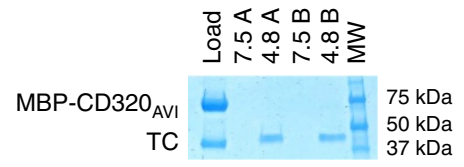

C

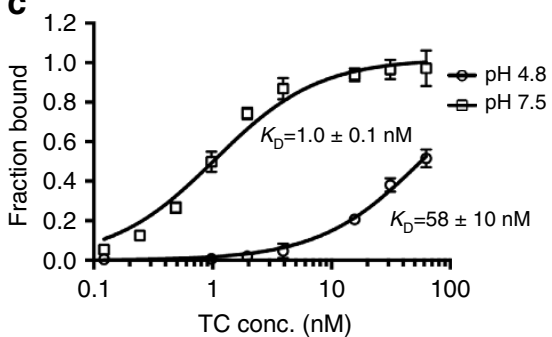

Figure 3 | Interface histidines and pH dependence of TC-CD320 affinity. (a) Histidine residues identified at the TC-CD320 interface: CD320 (yellow and gold) and TC (blue) are shown as backbone traces, with histidine residues at the binding interface shown as respectively coloured sticks. (b) SDS-polyacrylamide gel electrophoresis (SDS-PAGE) showing TC dissociation from an immobilized $\mathrm{CD}_{2} 2 \mathrm{O}_{\mathrm{AVI}} \mathrm{TC}^{\mathrm{T}}$ complex at $\mathrm{pH} 4.8$ but not at pH 7.5. Two successive washes (A and B) are shown. (c) TC-CD320 binding curves at pH 4.8 and 7.5 obtained from solid-phase binding experiments $(n=5$, error bars indicate s.d.).

and we observed no significant changes in TC or the LDLR-A2 domain, ruling out a gross structural effect of the $\Delta \mathrm{E} 88$ mutation. As seen for the wild-type protein, the region of LDLR-A1 carrying the deletion (EEE loop) revealed poor electron density and higher $\mathrm{B}$-factors when compared with that of LDLR-A2 (average B-factors for LDLR-A1 and $\mathrm{A} 2 \mathrm{Ca}^{2+}$ ions $\sim 112$ and $56 \AA^{2}$, respectively). Moreover, electron density for the $\mathrm{Ca}^{2+}$ binding residue Glu86 as well as Glu87 and disulfide forming Cys88 (equivalent to Cys89 in wild type) was missing. However, the centrally bound $\mathrm{Ca}^{2+}$ as well as the LDLR-A1 acidic necklace was clearly visible in our structure indicating intact $\mathrm{Ca}^{2+}$ binding. Even though the apparent Tm of the complex was slightly lower than that of the wild-type protein, approaching that of the LDRL-A2 domain alone (Supplementary Fig. 3b), the mutant protein bound TC with equivalent affinity to wild type with a calculated $K_{\mathrm{D}}$ of $1.5 \mathrm{nM}$ (Supplementary Fig 3c).

Role of the EGF domain of CD320 in TC binding. The exact function of the EGF-like domain in CD320 has not yet been established and its deletion in CD320 was shown to have no effect on TC binding 8 . The EGF-like domain of CD320 is not visible in our electron density maps despite chemically being present, suggesting positional disorder in the crystal. To assess what effect the domain has on CD320 complex formation with TC, we generated a mutant of CD320 in which the EGF-like domain was replaced by $\left(\mathrm{Gly}_{4} \mathrm{Ser}\right)_{4}$ sequence, which constituted a linker long enough to offer unrestricted movement and flexibility to the LDLR-A modules that the disulfide-rich EGF-like domain would not allow. The mutant protein was co-purified with TC and appeared biochemically stable and capable of TC binding. However, we found that the resulting construct yielded additional species of higher molecular weight in SEC in addition to the 1:1 stoichiometry complex peak seen for wild type (Fig. 4a). As shown in Fig. $4 \mathrm{~b}$, these results were confirmed by native electrospray ionization mass spectrometry analysis of the TC-CD320(Gly $\left.{ }_{4} \mathrm{Ser}\right)_{4}$ complex as well as its individually purified SEC peaks. We observed single species of $\sim 120 \mathrm{kDa}$ mass for the larger SEC peak and $65 \mathrm{kDa}$ mass for the smaller SEC peak, which we interpret as 2:1 or 2:2 TC:CD320 species and a single 1:1 complex, respectively.

\section{Discussion}

Our structure offers a basis for the selectivity of CD320 for TC over the homologous and structurally similar HC. This is especially relevant in light of HC's suggested role as a scavenger protein for corrin-like substrates in the blood stream that could otherwise bind TC and enter the cell and interfere with downstream Cbl metabolism. Unlike TC, both IF and HC are glycoproteins and it was previously suggested that $\mathrm{N}$-linked glycosylation of surface asparagines of $\mathrm{HC}$ might prevent its binding to CD320 (ref. 21). A comparison of receptor-bound TC with the recently determined HC structure (Fig. 5a) rules out such an explanation, as the glycans are not located at the appropriate surface. Rather, TC and HC have different electrostatic potentials at the relevant surfaces (Fig. 5b), with Lys105 and Lys114 in TC replaced by Asp92 and Asn102 in HC, respectively. The resultant electrostatic mismatch is especially pronounced for regions of HC that would contact LDLR-A2, which is the major determinant of stable complex formation. Thus, HC cannot bind CD320 due to electrostatic repulsion

The finding that the TC-CD320 interaction only involves the alpha domain of TC is in contrast to the recognition interface of IF with Cubilin, where both the $\alpha$ and $\beta$ domains of holo-IF are involved $^{19,41}$. Our findings suggest that in the absence of gross structural changes in the $\alpha$ domain on loss of bound $\mathrm{Cbl}$, both holo and apo-TC should be able to interact with CD320. Earlier studies have indeed pointed to receptor recognition of apo-TC, although a higher affinity was reported for holo-TC (refs 42-44). The TC-CD320 binding interface is only slightly larger than that of the LDLR-RAP complex (buried surface area $\sim 760 \AA^{2}$ ) and whereas the two LDLR-A modules in RAP contribute equally to the buried surface area and use virtually identical modes of ligand recognition, LDLR-A2 in CD320 serves as the primary determinant of high affinity binding. LDLR-A1, which displays low in vitro affinity to TC on its own, probably has a role in enhancing the specificity and affinity of CD320 for TC. The mutant CD320 $\mathrm{E} 88$, although more prone to forming non-specific complexes with TC (Supplementary Fig. 4), displays high thermal stability as well as tight TC binding. At least in vitro, these findings rule out a compromised ability of CD320 $\Delta \mathrm{E} 88$ to bind TC. Additional studies are needed to explain the in vivo effect of the mutation on receptor regulation and trafficking. Our data also support a structural role of the EGF-like domain of CD320 in influencing the flexibility and the orientation of 
a

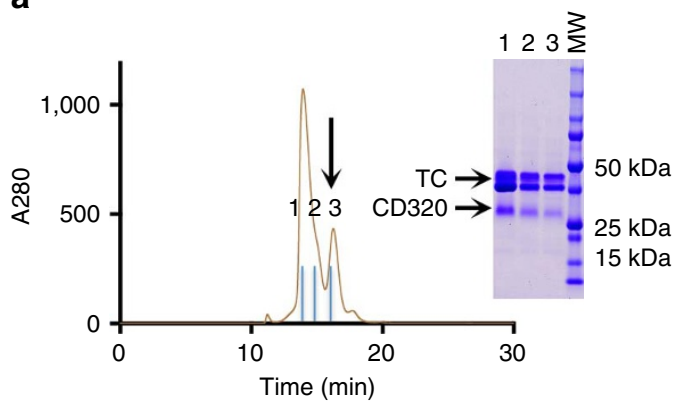

C

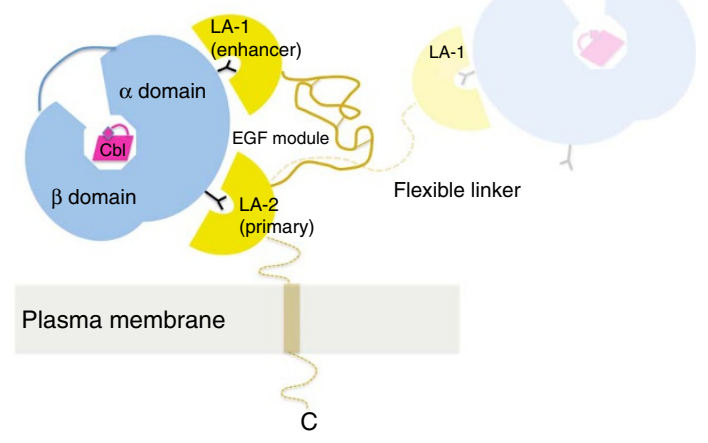

b
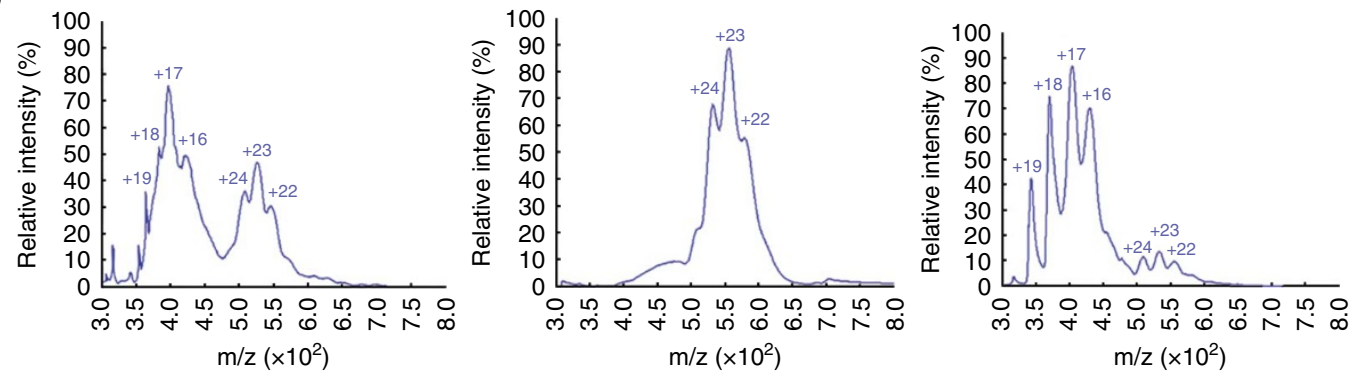

Figure 4 | Role of EGF-like domain in TC-CD320 complex formation. (a) SEC profile of TC-CD320(Gly Ser $_{4}$ construct, revealing higher mass peaks indicating multimeric species. Arrow indicates position of 1:1 TC-CD320 peak. Peak fractions indicated above SEC profile correspond to lanes in SDSpolyacrylamide gel electrophoresis (SDS-PAGE). Note that TC appears as a double band depending on polyacrylamide percentage and SDS-PAGE running conditions. (b) Native electrospray ionization mass spectrometry (ESI-MS) analysis of TC-CD320(Gly 4 Ser) ${ }_{4}$ complex. Each peak cluster comprises different charged species of a single macromolecular assembly formed due to the presence of residual salt from protein purification and correspond to approximate molecular weights (MWs) of 65 and 120 for the lower and higher molecular weight species, respectively (left panel). Analysis of Individually purified SEC peaks shows isolated high MW (middle) and low MW (right) species. (c) Model of properly oriented LDLR-A1 and LDLR-A2 domains (strong yellow) acting in tandem to specifically bind a single TC molecule. Dashed line indicates a flexible linker with proposed second TC molecule (faded yellow) binding.

the two LDLR-A domains to ensure that both LDLR-A domains bind to a single TC (Fig. 4c), thereby preventing formation of non-specific multimers that could potentially be detrimental to proper ligand internalization.

The combination of an acidic environment and decreased $\mathrm{Ca}^{2+}$ concentration has been implicated in the release of ligands of LDLRs during endocytosis (as recently reviewed ${ }^{45}$ ). However, no direct demonstration of $\mathrm{pH}$-mediated dissociation of the TC-CD320 complex was reported. Our in vitro finding that the affinity of TC for CD320 is reduced more than 50 -fold at low $\mathrm{pH}$ is in line with the expected receptor-ligand segregation and TC delivery to the lysosomal pathway. Our in vitro system sets the stage for addressing more detailed questions in future studies. These include whether acidification promotes $\mathrm{Ca}^{2+}$ release from the LDLR-A domains, how CD320 changes at low pHs, and whether such changes alter the ability of $\mathrm{CD} 320$ to bind potential regulators.

Finally, the ability to produce a stable and intact TC-CD320 complex, along with knowledge of the binding interface at atomic detail, might facilitate the design and manufacture of small molecule as well as immunotherapeutic regulators that could selectively disrupt the interaction in certain tumours overexpressing CD320. As for the latter, we have put in significant effort towards and been successful in the generation of camelid nanobodies against the TC-CD320 complex targeting various epitopes. These have the potential not only to aid in capturing novel conformational states of CD320 for structure determination, but also add to list of potential tools available for diagnostic or therapeutic applications in cancer treatments.

\section{Methods}

Protein expression and purification. The gene for human TC harbouring a R209Q mutation (GenBank ID BC001176.1) was obtained from ImaGenes (Life sciences). All CD320 constructs were $\mathrm{N}$-terminally fused to a nona-histidine tagged MBP construct with a tobacco etch virus (TEV) protease cleavage sequence inserted between the MBP and receptor genes. The native secretion signals for both CD320 and TC constructs were replaced with a Melittin secretion signal for enhanced secreted expression in Spodoptera frugiperda (Sf21 cells) using the Multibac expression system ${ }^{46}$. CD320 and TC constructs were cloned into the dual expression pFL vector and bacmids, and viruses were generated according to recommended procedures. For CD320, residues 31-199 and 53-199 were used. LDLR-A1 and LDLR-A2 constructs comprised residues 53-95 and 125-199, respectively. The TC $\alpha$ construct comprised residues 1-290 of TC. Sf21 cells were grown and maintained in SF900-II medium and protein was expressed for $96 \mathrm{~h}$ post infection. Bulk medium was then harvested, supplemented with $1 \mu \mathrm{M} \mathrm{Cbl-CN}$, and its $\mathrm{pH}$ was adjusted to 6.5 before being applied to Ni-NTA resin equilibrated in $20 \mathrm{mM}$ HEPES pH 7.0, $0.5 \mathrm{mM} \mathrm{CaCl}_{2}$ and $150 \mathrm{mM} \mathrm{NaCl}$. The resin was washed with $20 \mathrm{mM}$ HEPES pH 7.0, $300 \mathrm{mM} \mathrm{NaCl}$, and $40 \mathrm{mM}$ imidazole and the protein eluted with $20 \mathrm{mM}$ HEPES pH 7.0, $150 \mathrm{mM} \mathrm{NaCl}, 0.5 \mathrm{mM} \mathrm{CaCl}_{2}$ and $200 \mathrm{mM}$ imidazole buffer. Eluted protein was desalted in $20 \mathrm{mM}$ Tris pH 7.5, $0.5 \mathrm{mM} \mathrm{CaCl}_{2}$ and $150 \mathrm{mM} \mathrm{NaCl}$ (Buffer A) before overnight cleavage of the 9xHis-MBP module with His-tagged TEV protease (prepared in-house) at a w/w ratio of 1:10 (TEV:CD320). The resultant TC-CD320 complex displayed weak affinity for $\mathrm{Ni}^{2+}$-NTA and could be purified from excess receptor through a second $\mathrm{Ni}^{2+}$ affinity purification step with excess receptor separated in the flow through and the intact receptor eluting with $20 \mathrm{mM}$ Imidazole. Final samples were desalted into Buffer A, concentrated to $10-20 \mathrm{mg} \mathrm{ml}^{-1}$ and flash frozen in liquid $\mathrm{N}_{2}$. The flexible linker mutant of CD320 was generated by replacing residues $96-135$ of CD320 with the eicosapeptide $\left(\mathrm{Gly}_{4} \mathrm{Ser}\right)_{4}$. Protein expression was performed as for wild-type TC-CD320. Where required, CD320 and its variants were individually expressed without TC in the same cloning/expression system.

Biotinylated receptor was prepared by adding a C-terminal Avi Tag (GLNDIFEAQKIEWHE) to CD320 and its variants. Expression and purification was identical to the wild-type complex except that no MBP cleavage was performed. Overnight biotinylation with the biotin ligase BirA (prepared in-house) was performed by adding 5-10 ug BirA per mg purified receptor in Buffer A 
a

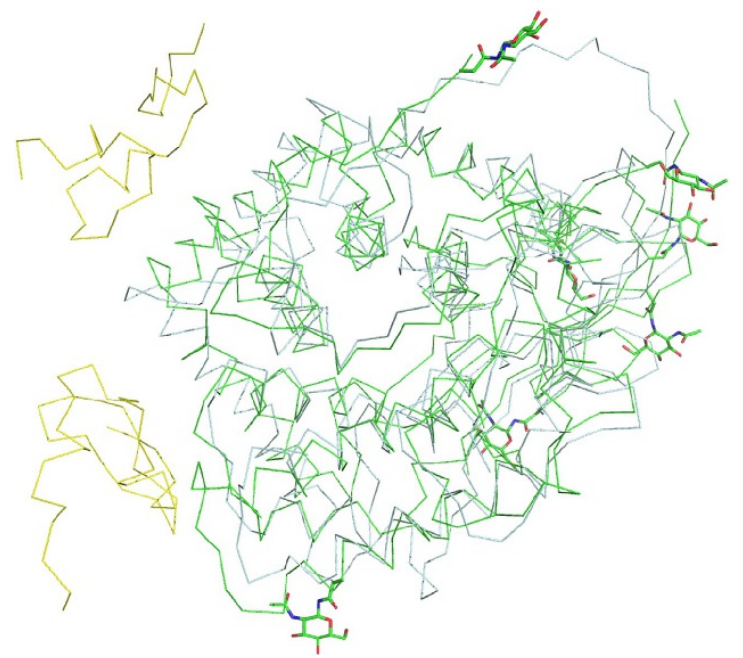

b
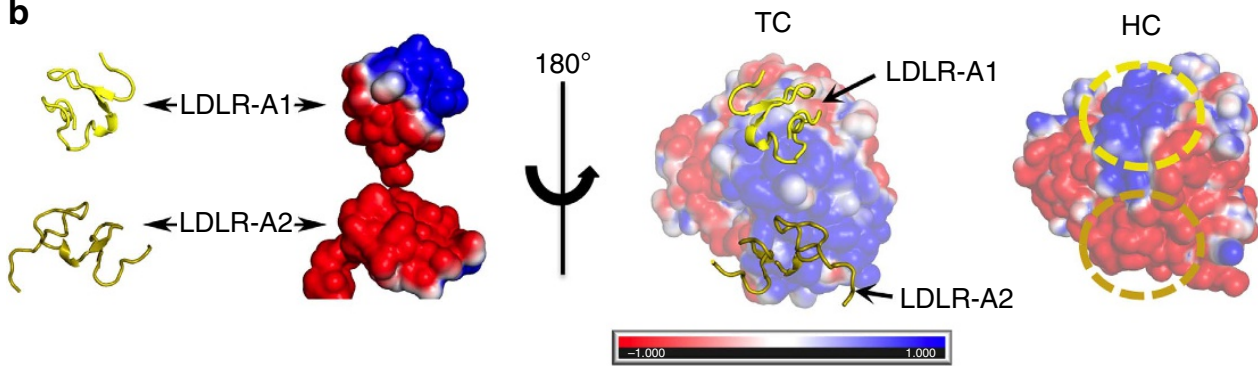

Figure 5 | Comparison of CD320-bound TC with free HC (pdb ID 4KKI). (a) Superposition of HC with CD320-bound TC revealing position of glycosylated residues (green sticks) of HC. (b) Electrostatic potential maps of CD320 (2nd from left), TC (2nd from right) and HC (right) with the latter two rotated $180^{\circ}$ along the $y$ axis in relation to CD320 to reveal the receptor interaction surfaces. For clarity, CD320 LDLR-A1 (yellow) and LDLR-2 (gold) are also show in cartoon format. The regions of HC corresponding to equivalent TC binding regions for LDLR-A1 and LDLR-A2 are highlighted (dotted yellow and gold ovals, respectively).

containing $10 \mathrm{mM}$ magnesium acetate, $10 \mathrm{mM}$ ATP and $50 \mu \mathrm{M}$ biotin. Biotinylation was confirmed by gel shift analysis on mixing desalted, biotinylated samples with streptavidin before SDS-polyacrylamide gel electrophoresis.

TC binding assay. Tag-less TC was obtained by dissociating a complex of TC and biotinylated CD320 immobilized on Streptactin resin (IBA) with four column volumes of Buffer B (Buffer A lacking $\mathrm{Ca}^{2+}$ and containing $10 \mathrm{mM}$ EGTA) at $37^{\circ} \mathrm{C}$ for $30 \mathrm{~min}$. The flow through containing TC was collected and the process repeated an additional three times. The pooled flow through samples were concentrated in an Amicon filter (30 kDa MWCO) and desalted in Buffer A. Protein concentrations were determined using a Bio-Rad DC Protein Assay Kit and $\mathrm{A}_{280}$ measurements.1-5 pMol receptor was added to each well of pre-blocked 96-well Neutravidin plates (ThermoFischer Scientific) for $1 \mathrm{~h}$ at room temperature (RT). All incubations were carried out on a plate shaker at 350 r.p.m. Unbound receptor was discarded and the wells were washed three times with $200 \mu \mathrm{l}$ Buffer A containing $0.05 \%$ Tween- 20 (Buffer AT). TC (100 ul) serially diluted in Buffer AT was added to the wells for $1 \mathrm{~h}$ at RT. Unbound TC was discarded and the wells washed five times with $200 \mu \mathrm{l}$ Buffer AT. To analyse binding at different pHs, Buffer A was modified to contain $100 \mathrm{mM}$ Tris- $\mathrm{HCl} \mathrm{pH} 7.5$ or $100 \mathrm{mM}$ sodium acetate $\mathrm{pH} 4.8$ instead of $20 \mathrm{mM}$ Tris- $\mathrm{HCl} \mathrm{pH} 7.5$ and used for serially diluting TC. An additional wash step in TC dilution buffer was added to equilibrate the wells before TC binding as well as after discarding unbound TC. TC antibody A-5 (Santa Cruz Biotechnology, Inc, Catalogue \# sc-137017) was diluted to $1 \mu \mathrm{g} \mathrm{ml}{ }^{-1}$ (1:200) in Buffer AT and $100 \mu \mathrm{l}$ was added to the wells for $30 \mathrm{~min}$ at RT. Unbound antibody was discarded and the wells washed three times with Buffer AT. Horse radish peroxidase $(100 \mu \mathrm{l})$ conjugated Goat mouse Anti IGg (ThermoFischer Scientific, Catalogue \# A16072) diluted to $1 \mu \mathrm{g} \mathrm{ml}^{-1}(1: 1,500)$ in Buffer AT was added to the wells for $30 \mathrm{~min}$. Unbound antibody was discarded and the wells washed three times with Buffer AT followed by development with the TMB Substrate Kit (ThermoFischer Scientific). Absorbance ( $450 \mathrm{nM})$ measurements were made with a BioTek Synergy HT plate reader. Absorbance readings were plotted against TC concentrations and curves fitted in GraphPad PRISM using a single-site specific binding equation, and normalized to the Calculated Bmax for direct comparison. All experiments were done in replicates of 5 .
Thermostability assays. Frozen protein samples were thawed and diluted to $0.5-1 \mathrm{mg} \mathrm{ml}^{-1}$ in Buffer A. A thermocycler (Bio-rad) was used to heat $80-100 \mathrm{ul}$ samples at the selected temperatures for $10 \mathrm{~min}$ followed by rapid cooling to $4{ }^{\circ} \mathrm{C}$ before spinning down at $120,000 \mathrm{~g}$ for $20 \mathrm{~min}$. Samples were loaded onto a G3000Swxl (TOSOH Biosciences) column pre-equilibrated in Buffer A at $4{ }^{\circ} \mathrm{C}$. Thermostability curves were plotted in GraphPad PRISM based on complex peak heights and fitted using a sigmoidal dose response (variable slope) equation. Protein used for assays in the presence of EGTA was diluted $20 \times$ to $0.5-1 \mathrm{mg} \mathrm{ml}^{-1}$ in Buffer B, which was also used for SEC.

Chemical Cross-linking. The protein complexes at $10 \mathrm{mg} \mathrm{ml}^{-1}$ were mixed with a $10 \%$ glutaraldehyde solution in a 10/1 (v/v) ratio for one hour at RT in buffer solutions. The mixture was further diluted with the original protein buffer solution or water before mass spectrometric analysis.

Mass spectrometry. A commercial MALDI-TOF/TOF mass spectrometer (model 4800 plus, AB Sciex, Darmstadt, Germany) equipped with a high-mass detector (HM2, CovalX AG, Zurich, Switzerland) was used. All measurements were performed in the linear positive ion mode with standard settings. Ionization was achieved with an Nd:YAG laser $(355 \mathrm{~nm})$ with the energy just above the threshold for ion formation. Each mass spectrum was the average of 1,000 laser shots acquired at random sample positions without searching for hot spot. Sinapinic acid (20 mg ml ${ }^{-1}$ in water/acetonitrile/TFA, 49.95/49.95/0.1, v/v/v) was used as the matrix. Matrix $(0.5 \mu \mathrm{l})$ was first spotted on a stainless steel plate and allowed to dry under ambient conditions. Afterwards, $0.5 \mu \mathrm{l}$ of the sample was spotted on the top of the matrix and also dried under ambient conditions. In the final step, $0.5 \mu \mathrm{l}$ of the matrix was spotted. All mass spectra were baseline corrected and smoothed using a Savitzky-Golay algorithm by Igor Pro 6.2, WaveMetrics, Oregon, USA.

Native ESI-mass spectrometry was carried out on a commercial hybrid quadrupole/time-of-flight mass spectrometer Synapt G2-S equipped with a Z-spray interface followed by the StepWave ion guides (Waters, Manchester, UK). The instrument was operated in the Resolution mode (resolution $>20,000$ ) in positive ion mode. For calibration, CsI clusters $\left(10 \mathrm{mg} \mathrm{ml}^{-1}\right)$ dissolved in 50\% (v) isopropanol was used. The recorded spectra were averaged (500 scans), smoothed with a moving 
algorithm (width of three steps) and centroid spectra were generated at $80 \%$ peak height. After polynomial fitting, the mass axis was calibrated. Typically, 3-5 $\mu$ of sample were loaded into $1 \mu \mathrm{m} \mathrm{Au} / \mathrm{Pd}$ coated glass needles (Thermo Scientific, Madison, WI, USA). The source temperature was kept at $30^{\circ} \mathrm{C}$. The backing pressure was set to 0.5 bar to assist sample flow. The capillary voltage was set to $0.95 \mathrm{~V}$ to generate the electrospray. The sampling cone voltage and the source offset were optimized to 150 and $50 \mathrm{~V}$ respectively, to ensure efficient ion transmission. Further, to assure better declustering of salt adducts, the trap collision energy and the transfer collision energy were kept at 30 and $15 \mathrm{~V}$, respectively. The quadrupole transmission range was adjusted for the desired $\mathrm{m} / \mathrm{z}$ range. The mass spectra were recorded in a $\mathrm{m} / \mathrm{z}$ window of $50-8,000$ with a scan time of $2 \mathrm{~s}$ and an interscan delay of $0.1 \mathrm{~s}$. Each native electrospray ionization mass spectrometry spectrum represents the combination of 150 individual scans. Mass spectra were acquired using Mass Lynx software (version 4.1, Waters, Manchester, UK). All mass spectra were baseline corrected, normalized and smoothed using MATLAB_R2014a.

Crystallization and structure determination. For crystallization of the wild-type TC-CD320 complex, frozen samples were thawed and diluted to $10 \mathrm{mg} \mathrm{ml}^{-1}$ and mixed with well buffer $\left(9.6 \%\right.$ PEG8000, $160 \mathrm{mM} \mathrm{Ca}(\mathrm{OAc})_{2}, 80 \mathrm{mM} \mathrm{MES}$ pH 6.2, and $20 \mathrm{mM} \mathrm{MgCl} 2$ ) in a $1: 1$ ratio at $20^{\circ} \mathrm{C}$ in sitting drop plates. Crystals were cryo-protected by exchanging the drop solution with 9.6\% PEG8000, $160 \mathrm{mM}$ $\mathrm{Ca}(\mathrm{OAc})_{2}, 80 \mathrm{mM}$ MES pH 6.2 , and $20 \mathrm{mM} \mathrm{MgCl}_{2} 20 \mathrm{mM}$ Tris $\mathrm{pH} 7.5$, $150 \mathrm{mM} \mathrm{NaCl}, 0.5 \mathrm{mM} \mathrm{CaCl} 2,1 \mu \mathrm{M} \mathrm{Cbl}-\mathrm{CN}$ and $25 \%$ Glycerol and flash frozen in liquid Nitrogen. An N-terminal 22 amino acid deletion of the receptor led to higher quality crystals that were used for the final structure determination.

Crystals of the $\Delta \mathrm{E} 88$ mutant were obtained by purifying the complex by SEC in Buffer A before concentrating to $10 \mathrm{mg} \mathrm{m}^{-1}$ and mixing with well buffer (180 mM L-Proline, 11.7\% PEG3350, $90 \mathrm{mM}$ Hepes pH 7.5, and $30 \mathrm{mM}$

Glycyl-Glycyl-Glycine) in a 2:1 ratio at $20^{\circ} \mathrm{C}$ in sitting drop plates. Crystals were cryo-protected by exchanging the drop solution with Cryo-buffer $(108 \mathrm{mM}$ L-Proline, $54 \mathrm{mM}$ Hepes pH 7.5, 7.2\% PEG3350, $0.6 \mathrm{mM} \mathrm{CaCl}_{2}, 24 \mathrm{mM}$ Tris $\mathrm{pH}$ $7.5,180 \mathrm{mM} \mathrm{NaCl}, 1 \mu \mathrm{M} \mathrm{Cbl}-\mathrm{CN}$ and $25 \%$ Glycerol) and flash frozen in liquid Nitrogen. Data were collected at the X06SA microfocus beamline (Swiss Light Source) at a wavelength of $1 \AA$. A complete data set from a single wild-type crystal was collected in six discreet wedges and processed using denzo and scalepack (HKL Research). The structure was determined by molecular replacement using PHASER in PHENIX (ref. 47) using a TC monomer from the Cbl- $\mathrm{H}_{2} \mathrm{O}$ bound human TC structure (PDB 2BB5) as a search model. Model building and refinement were carried out using COOT (ref. 48) and PHENIX. Anomalous data sets from an identical crystal collected at $1.6 \AA$ wavelength was processed by denzo and scalepack. Two molecules of the TC-CD320 complex/asymmetric unit were found, molecule 1 comprising chains $\mathrm{A}$ and $\mathrm{C}$ and molecule 2 comprising chains $\mathrm{B}$ and $\mathrm{D}$.

A complete data set for the $\triangle \mathrm{E} 88$ mutant was processed by XDS (ref. 49) and aimless in CCP4 (ref. 50). The wild-type TC-CD320 structure was used as a search model for molecular replacement using PHASER in PHENIX. Model building and refinement were carried out using COOT and PHENIX. An anomalous data set collected at $1.77 \AA$ wavelength was processed by XDS and aimless in CCP4. TC-CD320 complex molecule 2 was used to generate all figures using the PyMOL Molecular Graphics System (The PyMOL Molecular Graphics System, Version 1.8 Schrödinger, LLC). Part of the $2 \mathrm{fo}-\mathrm{fc}$ electron density maps for both the wild-type and CD320 $\mathrm{E} 88$ complexes with TC are shown in Supplementary Fig. 6.

Data availability. Atomic structure factors and coordinates for the TC-CD320 and TC-CD320 $\Delta$ E88 structures have been deposited under accession codes 4ZRP and $4 \mathrm{ZRQ}$, respectively. The authors declare that all relevant data sets used in this study are available on request.

\section{References}

1. Cannata, J. J., Focesi, Jr A., Mazumder, R., Warner, R. C. \& Ochoa, S. Metabolism of propionic acid in animal tissues. Xii. Properties of mammalian methylmalonyl coenzyme a mutase. J. Biol. Chem. 240, 3249-3257 (1965).

2. Taylor, R. T. \& Weissbach, H. N5-methyltetrahydrofolate-homocysteine transmethylase. Propylation characteristics with the use of a chemical reducing system and purified enzyme. J. Biol. Chem. 242, 1509-1516 (1967).

3. Johnston, J., Bollekens, J., Allen, R. H. \& Berliner, N. Structure of the cDNA encoding transcobalamin I, a neutrophil granule protein. J. Biol. Chem. 264, 15754-15757 (1989).

4. Allen, R. H. Human vitamin B12 transport proteins. Prog. Hematol. 9, 57-84 (1975).

5. Birn, H. et al. Characterization of an epithelial approximately $460-\mathrm{kDa}$ protein that facilitates endocytosis of intrinsic factor-vitamin B12 and binds receptor-associated protein. J. Biol. Chem. 272, 26497-26504 (1997).

6. Kozyraki, R. et al. The human intrinsic factor-vitamin B12 receptor, cubilin: molecular characterization and chromosomal mapping of the gene to $10 \mathrm{p}$ within the autosomal recessive megaloblastic anemia (MGA1) region. Blood 91, 3593-3600 (1998).
7. Quadros, E. V., Sai, P. \& Rothenberg, S. P. Characterization of the human placental membrane receptor for transcobalamin II-cobalamin. Arch. Biochem. Biophys. 308, 192-199 (1994).

8. Jiang, W., Nakayama, Y., Sequeira, J. M. \& Quadros, E. V. Mapping the functional domains of TCblR/CD320, the receptor for cellular uptake of transcobalamin-bound cobalamin. FASEB J. 27, 2988-2994 (2013).

9. Chanarin, I., Muir, M., Hughes, A. \& Hoffbrand, A. V. Evidence for intestinal origin of transcobalamin II during vitamin B12 absorption. Br. Med. J. 1, 1453-1455 (1978).

10. Nielsen, M. J., Rasmussen, M. R., Andersen, C. B., Nexo, E. \& Moestrup, S. K. Vitamin B12 transport from food to the body's cells--a sophisticated, multistep pathway. Nat. Rev. Gastroenterol. Hepatol. 9, 345-354 (2012).

11. Reynolds, E. Vitamin B12, folic acid, and the nervous system. Lancet Neurol. 5, 949-960 (2006).

12. Kozyraki, R. \& Cases, O. Vitamin B12 absorption: mammalian physiology and acquired and inherited disorders. Biochimie 95, 1002-1007 (2013).

13. Quadros, E. V., Nakayama, Y. \& Sequeira, J. M. The protein and the gene encoding the receptor for the cellular uptake of transcobalamin-bound cobalamin. Blood 113, 186-192 (2009).

14. Quadros, E. V. Advances in the understanding of cobalamin assimilation and metabolism. Br. J. Haematol. 148, 195-204 (2010).

15. Brown, M. S. \& Goldstein, J. L. A receptor-mediated pathway for cholesterol homeostasis. Science 232, 34-47 (1986)

16. Blacklow, S. C. Versatility in ligand recognition by LDL receptor family proteins: advances and frontiers. Curr. Opin. Struct. Biol. 17, 419-426 (2007).

17. Hussain, M. M., Strickland, D. K. \& Bakillah, A. The mammalian low-density lipoprotein receptor family. Annu. Rev. Nutr. 19, 141-172 (1999).

18. Willnow, T. E. et al. RAP, a specialized chaperone, prevents ligand-induced ER retention and degradation of LDL receptor-related endocytic receptors. $E M B O$ J. 15, 2632-2639 (1996).

19. Andersen, C. B., Madsen, M., Storm, T., Moestrup, S. K. \& Andersen, G. R. Structural basis for receptor recognition of vitamin-B(12)-intrinsic factor complexes. Nature 464, 445-448 (2010).

20. Wuerges, J. et al. Structural basis for mammalian vitamin B12 transport by transcobalamin. Proc. Natl Acad. Sci. USA 103, 4386-4391 (2006).

21. Wuerges, J., Geremia, S. \& Randaccio, L. Structural study on ligand specificity of human vitamin B12 transporters. Biochem. J. 403, 431-440 (2007).

22. Furger, E., Frei, D. C., Schibli, R., Fischer, E. \& Prota, A. E. Structural basis for universal corrinoid recognition by the cobalamin transport protein haptocorrin. J. Biol. Chem. 288, 25466-25476 (2013).

23. Quadros, E. V., Nakayama, Y. \& Sequeira, J. M. Targeted delivery of saporin toxin by monoclonal antibody to the transcobalamin receptor, TCblR/CD320. Mol. Cancer Ther. 9, 3033-3040 (2010)

24. Quadros, E. V., Nakayama, Y. \& Sequeira, J. M. Saporin conjugated monoclonal antibody to the transcobalamin receptor TCblR/320 is effective in targeting and destroying cancer cells. J. Cancer Ther. 4, 1074-1081 (2013).

25. Quadros, E. V., Sai, P. \& Rothenberg, S. P. Functional human transcobalamin II isoproteins are secreted by insect cells using the baculovirus expression system. Blood 81, 1239-1245 (1993).

26. Seligman, P. A. \& Allen, R. H. Characterization of the receptor for transcobalamin II isolated from human placenta. J. Biol. Chem. 253, 1766-1772 (1978).

27. Yasui, N., Nogi, T. \& Takagi, J. Structural basis for specific recognition of reelin by its receptors. Structure 18, 320-331 (2010).

28. Fedosov, S. N. et al. Mapping the functional domains of human transcobalamin using monoclonal antibodies. FEBS J. 272, 3887-3898 (2005).

29. Fedosov, S. N., Fedosova, N. U., Nexo, E. \& Petersen, T. E. Conformational changes of transcobalamin induced by aquocobalamin binding. Mechanism of substitution of the cobalt-coordinated group in the bound ligand. J. Biol. Chem. 275, 11791-11798 (2000).

30. Fass, D., Blacklow, S., Kim, P. S. \& Berger, J. M. Molecular basis of familial hypercholesterolaemia from structure of LDL receptor module. Nature $\mathbf{3 8 8}$, 691-693 (1997).

31. Rudenko, G. et al. Structure of the LDL receptor extracellular domain at endosomal pH. Science 298, 2353-2358 (2002).

32. Fisher, C., Beglova, N. \& Blacklow, S. C. Structure of an LDLR-RAP complex reveals a general mode for ligand recognition by lipoprotein receptors. Mol. Cell 22, 277-283 (2006).

33. Beglov, D. et al. Structural insights into recognition of beta2-glycoprotein I by the lipoprotein receptors. Proteins 77, 940-949 (2009).

34. Verdaguer, N., Fita, I., Reithmayer, M., Moser, R. \& Blaas, D. X-ray structure of a minor group human rhinovirus bound to a fragment of its cellular receptor protein. Nat. Struct. Mol. Biol. 11, 429-434 (2004).

35. Kurniawan, N. D. et al. NMR structure of a concatemer of the first and second ligand-binding modules of the human low-density lipoprotein receptor. Protein Sci. 9, 1282-1293 (2000). 
36. Beglova, N., Jeon, H., Fisher, C. \& Blacklow, S. C. Cooperation between fixed and low $\mathrm{pH}$-inducible interfaces controls lipoprotein release by the LDL receptor. Mol. Cell 16, 281-292 (2004).

37. Beglova, N. \& Blacklow, S. C. The LDL receptor: how acid pulls the trigger. Trends Biochem. Sci. 30, 309-317 (2005).

38. Chung, N. S. \& Wasan, K. M. Potential role of the low-density lipoprotein receptor family as mediators of cellular drug uptake. Adv. Drug Deliv. Rev. 56, 1315-1334 (2004)

39. Yamamoto, T., Chen, H. C., Guigard, E., Kay, C. M. \& Ryan, R. O. Molecular studies of $\mathrm{pH}$-dependent ligand interactions with the low-density lipoprotein receptor. Biochemistry 47, 11647-11652 (2008).

40. Quadros, E. V. et al. Positive newborn screen for methylmalonic aciduria identifies the first mutation in TCblR/CD320, the gene for cellular uptake of transcobalamin-bound vitamin B(12). Hum. Mutat. 31, 924-929 (2010).

41. Fedosov, S. N. et al. Composite organization of the cobalamin binding and cubilin recognition sites of intrinsic factor. Biochemistry 44, 3604-3614 (2005).

42. Rothenberg, S. P. \& Quadros, E.V. Transcobalamin II and the membrane receptor for the transcobalamin II-cobalamin complex. Baillieres Clin. Haematol. 8, 499-514 (1995).

43. Quadros, E. V., Nakayama, Y. \& Sequeira, J. M. The binding properties of the human receptor for the cellular uptake of vitamin B12. Biochem. Biophys. Res. Commun. 327, 1006-1010 (2005).

44. Orning, L. et al. Characterization of a monoclonal antibody with specificity for holo-transcobalamin. Nutr. Metab. (Lond) 3, 3 (2006).

45. Andersen, C. B. \& Moestrup, S. K. How calcium makes endocytic receptors attractive. Trends Biochem. Sci. 39, 82-90 (2014).

46. Bieniossek, C., Richmond, T. J. \& Berger, I. MultiBac: multigene baculovirusbased eukaryotic protein complex production. Curr. Protoc. Protein Sci.. Chapter 5, Unit 520 (2008).

47. Adams, P. D. et al. PHENIX: a comprehensive Python-based system for macromolecular structure solution. Acta Crystallogr. D Biol. Crystallogr. 66, 213-221 (2010).

48. Emsley, P., Lohkamp, B., Scott, W. G. \& Cowtan, K. Features and development of Coot. Acta Crystallogr. D Biol. Crystallogr. 66, 486-501 (2010).

49. Kabsch, W. Xds. Acta Crystallogr. D Biol. Crystallogr. 66, 125-132 (2010).

50. Winn, M. D. et al. Overview of the CCP4 suite and current developments. Acta Crystallogr. D Biol. Crystallogr. 67, 235-242 (2011).

\section{Acknowledgements}

We gratefully acknowledge the assistance of staff scientists at the PX beamline of the Swiss Light Source, Villigen, Switzerland. This work was supported by the Swiss Cancer League (KFS 3004-08-2012 to K.P.L.), NCCR TransCure (grant to K.P.L.), the Swiss National Science Foundation (SNF 200020-124663 to R.Z. and SNF 31003A_146191 and SNF 310030B_166672 to K.P.L.) and the European Molecular Biology Organization (EMBO ALTF-770-2010 to A.A.)

\section{Author contributions}

A.A. carried out the structure determination of TC-CD320 complexes as well as all other experimental procedures excluding Mass Spectrometry analyses. J.-S.W. cloned and crystallized the initial TC-CD320 complexes. J.S. contributed to the cloning and purification of TC $\alpha$-CD320 and TC-LDLR-A2 complexes. B.P. helped with protein expression and purification experiments. J.S.B. generated expression plasmids and viruses for some constructs. F.C., K.R. and R.Z performed mass spectrometry analyses K.P.L., A.A., and J.-S.W. conceived the project. K.P.L. and A.A. analysed the data and wrote the manuscript.

\section{Additional information}

Supplementary Information accompanies this paper at http://www.nature.com/ naturecommunications

Competing financial interests: The authors declare no competing financial interests.

Reprints and permission information is available online at http://npg.nature.com/ reprintsandpermissions/

How to cite this article: Alam, A. et al. Structural Basis of Transcobalamin Recognition by human CD320 Receptor. Nat. Commun. 7:12100 doi: 10.1038/ncomms12100 (2016).

(c) (i) This work is licensed under a Creative Commons Attribution 4.0 International License. The images or other third party material in this article are included in the article's Creative Commons license, unless indicated otherwise in the credit line; if the material is not included under the Creative Commons license, users will need to obtain permission from the license holder to reproduce the material. To view a copy of this license, visit http://creativecommons.org/licenses/by/4.0/

(C) The Author(s) 2016 